\title{
Texture Image Analysis for Osteoporosis Detection with Morphological Tools
}

Sylvie Sevestre-Ghalila ${ }^{1}$, Amel Benazza-Benyahia ${ }^{2}$, Anne Ricordeau $^{3}$, Nedra Mellouli ${ }^{3}$, Christine Chappard ${ }^{4}$, and Claude Laurent Benhamou ${ }^{4}$

${ }^{1}$ Université Paris 5, Laboratoire MAP5, 45 rue des Saints Pères 75270 Paris Cédex 06, FRANCE - Université Manouba, ENSI, Laboratoire CRISTAL-GRIFT, campus de la Manouba, 2020 La Manouba, Tunisie. sevestre@math-info.univ-paris5.fr

${ }^{2}$ Ecole Supérieure des Communications de Tunis, URISA, Tunisia

${ }^{3}$ Université Paris 8, LINC, IUT de Montreuil, Montreuil, France

${ }^{4}$ CHR d'Orléans, France

\begin{abstract}
Osteoporosis is due to the following two phenomena: a reduction bone mass and a degradation of the microarchitecture of bone tissue. In this paper, we propose a method for extracting morphological information enabling the description of bone structure from radiological images of the calcaneus. Our main contribution relies on the fact that we provide bone descriptors close to classical 3D-morphological bone parameters. The first step of the proposed method consists in extracting the grey-scale skeleton of the microstructures contained in the underlying images. After an appropriate processing, the resulting skeleton provides discriminant features between osteoporotic patients and control patients. Statistical tests corroborate this discrimination property.
\end{abstract}

\section{Introduction}

Clinically, osteoporosis is a consequence of a gradual loss of calcium and collagen which induces both a dramatical bone mass decrease and, an alteration of the trabecular microarchitecture [1]. As the skeleton becomes too fragile to support the body, fractures occur frequently, especially in the wrist, spine or femur. In this context, diagnosing osteoporosis and assessing the efficacy of a treatment is a challenging issue for the health community. Since several years, various techniques have been developed in order to detect early and efficiently the apparition of this disease before fracture. The decrease of the Bone Mineral Density (BMD) is generally assessed through non-invasive methods as the dual energy X-ray absorptiometry. However, it has been recognized that in addition to BMD tests, information about the bone structure helps to determine the bone quality and hence, the probability of fractures [2]. Indeed, the bone structure consists of arches (trabeculae) that are arranged in groups according to different orientations that correspond mainly to compression/tensile stress. The analysis of such a stucture provides valuable information about the bone's mechanical strength and the alteration of its microarchitecture and hence, about the disease 
apparition or evolution. For instance, the bone is scanned by Magnetic Resonance Imaging (MRI) or X-rays in order to generate a textured "image" of the real bone structure. However, MRI suffers from a limited spatial resolution [3] and Computed Tomography (CT) techniques have limited applicability because of the high radiation dose. This is the reason why plane-film radiograph is preferred since it is inexpensive and requires much lower radiation doses. It is worth pointing out that the resulting $2 \mathrm{D}$ radiological patterns are the projection of the $3 \mathrm{D}$ anatomical structures on the plane film. Despite the artefacts inherent to the projection procedure, radiological trabecular patterns are assumed to reflect the anatomical one. The great irregularity (in shape, size and thickness) of the radiographic pattern and the low contrast in certain areas make difficult the interpretation of bone alteration by visual inspection. Therefore, computeraided methods of texture analysis have been developed to assist clinicians in their diagnosis. The most challenging task consists in characterizing the bone microarchitecture by parameters automatically estimated from the images that are able to accurately detect and quantify the alterations of the bone. . In this work, we are interested in detecting osteoporosis through a texture analysis of calcaneus radiographs. Indeed, calcaneus is known to contain rich information on microarchitectural patterns and the required image acquisition system can be easily adjusted [6]. Most methods for such detection task [4-8] use classical approaches in texture analysis domain. Related to the approach of Gereats[9], our contribution consists in using morphological tools in order to extract characteristic features of the trabecular bone image close to classical 3D-morphological parameters. This paper is organized as follows. In Section 2, the skeletonization procedure and its composition are briefly described. In Section 3 the problem of the skeleton characterization is addressed and skeleton features are introduced. In Section 4, some experimental results are reported and commented. Finally, in Section [5, some conclusions are drawn.

\section{Computing the Skeleton}

\section{$2.1 \quad$ Test Images}

First of all, some information about image acquisition is given. Radiographic film is used for the formation of each image; the region of interest is $2.7 \mathrm{~cm}$ $\times 2.7 \mathrm{~cm}$. This area only includes trabecular bone in the posterior part of the calcaneus and has a reproducible location [6]. The same X-ray clinical apparatus, with a tube voltage of $36 \mathrm{kV}$, with a $18 \mathrm{mAs}$ exposure level with a Min-R film is employed during the acquisition procedure. The radiographs are scanned by an Agfa duoscan enabling their digital conversion. The scanning resolution amounts $100 \mu \mathrm{m}$ so as a pixel represents an area of $105 \mu \mathrm{m} \times 105 \mu \mathrm{m}$. Finally, the resulting digital images $I(m, n)$ have a size of $256 \times 256$ and are coded at $8 \mathrm{bpp}$. A number of 31 images of Osteoporotic Patients (OP) with vetebral fractures and (or) with other kind of fractures and 39 images of Control Patients (CP) matched for age, are considered. 


\subsection{The Corresponding Skeleton}

During the first stage of processing, the aim was to extract the grey-level skeleton of the microstructures contained in the underlying images. The skeleton consists of a network of arches which are located along local higher intensity regions. Its extraction was not an easy task because of the lack of a unifying approach for grey-scale image skeletonization [10]11]. In our case, a meaningful skeleton of the microarchitectures was expected preserve the microarchitecture connectivity. Therefore, we applied the skeletonization algorithm described in [12 13. Indeed, the underlying thinning procedure is homotopic which is a crucial property for shape description applications. Furthermore, the skeletonization procedure had a very low computational cost. Figure 1.(b) provides an example of a resulting skeleton in the OP case. As can be seen, the skeleton corresponds to a very connected network of arches. The principal arches are vertical in the region of interest and originating in the subtalar joint, they correspond to the longitudinal trabeculae. The incidental arches are located in the bottom left of the region of interest originating in the inferior cortice of the calcaneus and they are associated with the transversal trabeculae.

Before characterizing the skeleton, it is necessary to determine its composition. Generally, a skeleton is viewed as the set of intersection points and the segments that connect such points. It is possible to classify the intersection pixels of the skeleton into the 2 following categories.

- The first one concerns pixels that are connected to at least 4 neighbors (according to the 8-connectivity). Since the maximum width of the skeleton amounts to 2 pixels, the pixels of this first class belong to intersection consisting of several pixels.

- The second category contains only pixels whose neighborhoods (according to the 8-connectivity) contain pixels that are not mutually 4-connected.

After determining the pixels of the first category, a label is assigned to each segment according to the segments emerging from each intersection. Indeed, at each intersection, the neighboring pixels (outside the intersection) are listed and if necessary, a new label is created for each of the listed pixels. This label is then propagated towards its nearest neighbor. It is worth noting that when the first pixel is receiving a label, two of its neighbors are potential candidates for the propagation procedure. The retained candidate is the neighbor which does not have any intersection pixel in its neighborhood. The scanning of the segment is stopped at a labeled pixel that encloses in its neighborhood an intersection pixel. The result of this classification is illustrated in the figure 1. (b). As a result, the projected portions of the trabeculae which correspond to the segments separating two adjacent multiple points can be identified.

\section{Exploiting the Skeleton}

The resulting grey-scale skeleton can be used to analyze the texture of the calcaneus. More precisely, we are interested here in quantifying the morphological features of the skeleton that give reliable information about the microarchitecture 


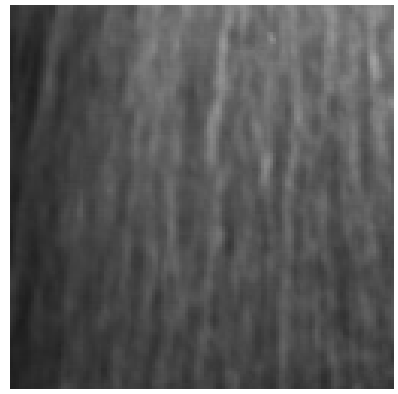

(a)

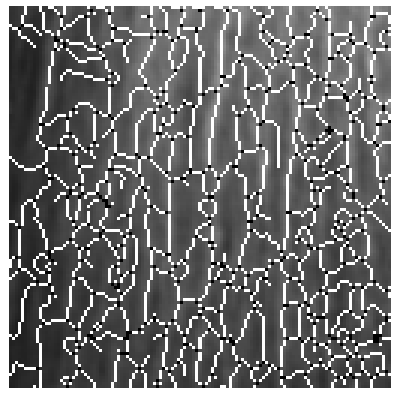

(b)

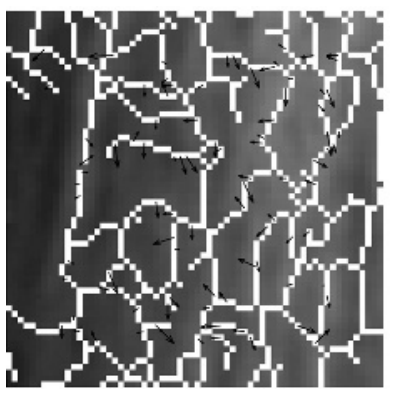

(c)

Fig. 1. Composition of skeleton: the image (b) is the pixel classification of the skeleton extrated from the image (a), the most black pixels are intersections. In the image (c) the half intra-projected distances of the left upper corner of (b) are illustrated with black arrows.

of the bone. The aim is to extract morphological features wich may be related to quantities obtained from invasive tests performed in pathological analysis.

\subsection{Inter-projected Distance}

An inter-projected distance is estimated as a length separating two successive intersections on a path of the skeleton, that is called a segment. The orientation of such a segment is measured with respect to the horizontal image axis. Again, for each segment, the orientation mean is defined as the average of the orientations calculated at each pixel of the underlying segment.

\subsection{Intra-projected Distance}

The intra-projected distances is the distances between the two lines of the maximum gradient directions around the skeleton. Hence, our objective is to determine for each segment point, the closest points to the left and the right of the segment where the absolute value of the gradient is maximum. Obviously, these points are searched along the normal to the segment [14]. The distance between the left or the right points is then computed and finally, the median value of these distances when the whole the segment is scanned could be considered as the half intra-projected distance. It is worth noting that this procedure is carried out only if the projected segment has three or more pixels, otherwise the derivative filters cannot be applied. Furthermore, the coherence between the direction of the skeleton and the direction of the detected border lines was controlled as in 14. Among 4000 segments are generally detected in an image. With these two contraints, about 1000 intra-projected distances were estimated by using only few pixels per segment. These estimations are depicted by the black arrows in Figure1. 


\subsection{Extracted Features}

As noted by radiologists, osteoporosis tends to alterate the trabeculae that are the least required during the body motion. In the case of calcaneus, these trabeculae correspond to transversal ones 15. Therefore, the features were separated into the two following categories :the first one is related to the transversal segments and corresponding to transversal trabeculae, the second related to the longitudinal segments corresponding to longitudinal trabeculae.

The histogram of the orientations of the segment is thresholded locally according to a sliding window. The largest value is associated with the longitudinal segments. The transversal and longitudinal projected segments do not share the same properties. To emphasize such dissymetry, the ratio between the variances of the angles and the ratio between the cardinalities of each class were evaluated. Within each class of orientation, we compute the following features :

- the mean $\bar{L}$ (resp. $\bar{l}$ ) of respectively the length $L$ and the half-width $l$ of the projected trabeculae; their variance $\sigma_{L}^{2}$ (resp. $\left.\sigma_{l}^{2}\right)$.

- the variance of the angles $\sigma^{2}(\theta)$ with respect to the local mean.

- the total number of pixels $N_{p}$ belonging to the skeleton.

- the total number of ending pixels $N_{e}$ of the skeleton.

\section{Experimental Results}

\subsection{Univariate Analysis}

In this part we evaluate de dispersion of each feature for CP and for OP class. The corresponding intervals mentioned in Table 1show OP has a larger variation than CP. Althougth the OP intervals overlap the CP ones, it can be noted that for a large number of skeleton features, CP and OP are significantly different. The exceptions are $N_{e}$ whatever the orientation, the mean length $(\bar{L})$ in the transverse direction, and the ratio between the values of $N_{p}$ in each direction. The $p$-values resulting from the Wilcoxon Test show that a better discrimination between $\mathrm{OP}$ and $\mathrm{CP}$ is obtained in the longitudinal direction. More precisely, it indicates that the inter-projected and intra-projected distances take globally highest values within the $\mathrm{CP}$ group. Indeed, the reduction of such distances, in the $\mathrm{OP}$ could be imputed to a greater transparency of the bone network due to the microarchitecture deterioration. More precisely, the transparency induced by the disease is generally the result of two phenomena : a thinning effect and a perforation effect which can quantified by $N_{e}$ feature.

\subsection{Multivariate Analysis}

In this section we are interesting to study the contribution of the morphological features combined with bone density (BMD) and Age, to discriminate between $\mathrm{OP}$ and $\mathrm{CP}$. To visualize multidimensionality, we use the principal components analysis (PCA) approach so as to illustrate the complementary 
Table 1. Statistical distributions of the considered parameters. The first table gives statistics on our features for the longitudinal direction and the second table for the transversal direction. For each direction, the associated table shows, for the length $\bar{L}$ respectively for the half-width $\bar{l}$ of the image, its interval of dispersion, its variance $\sigma_{L}^{2}\left(\operatorname{resp} . \sigma_{l}^{2}\right)$, the total number of pixels $N_{p}$ belonging to the skeleton and the total number of ending segments $N_{e}$ of the skeleton. The third table gives global statistics of the skeleton for $\mathrm{OP}$ and $\mathrm{CP}$ as the total pixels of the skeleton $N_{p}$, the total number of intersections, the rate of the total number of pixels in longitudinal and transversal direction $N_{p}^{\mathrm{L}} / N_{p}^{\mathrm{T}}$, and the rate of the variance of the angles $\sigma^{2}(\theta)$ with respect to the local mean of each direction.

\begin{tabular}{|c|c|c|c|c|c|c|c|}
\hline Longitudinal & \multicolumn{2}{|c|}{$L$} & l & $\sigma_{L}$ & $\sigma_{l}$ & $N_{p}$ & $N_{e}$ \\
\hline Osteoporotic & \multicolumn{2}{|c|}{$\left[\begin{array}{lll}3.61 & 5.56\end{array}\right]$} & {$\left[\begin{array}{lll}0.29 & 1.02\end{array}\right]$} & {$\left[\begin{array}{ll}1.01 & 2.89\end{array}\right]$} & {$\left[\begin{array}{ll}0.65 & 1.15\end{array}\right]$} & {$\left[\begin{array}{lll}4078 & 5800\end{array}\right]$} & $\leq 110]$ \\
\hline Control & \multicolumn{2}{|c|}{$\left[\begin{array}{lll}4.16 & 5.12\end{array}\right]$} & $\begin{array}{lll}0.49 & 0.95 \\
\end{array}$ & {$\left[\begin{array}{ll}1.44 & 2.60\end{array}\right]$} & {$\left[\begin{array}{lll}0.75 & 1.14\end{array}\right]$} & {$\left[\begin{array}{lll}4756 & 5534\end{array}\right]$} & {$\left[\begin{array}{ll}33 & 69\end{array}\right]$} \\
\hline $\begin{array}{c}\text { Wilcoxon Test } \\
(\mathrm{p} \text {-value })\end{array}$ & \multicolumn{2}{|c|}{$\begin{array}{l}C>O \\
(0.004)\end{array}$} & $\begin{array}{c}C>O \\
(0.0056)\end{array}$ & $\begin{array}{c}C>O \\
(0.0054)\end{array}$ & $\begin{array}{l}C>O \\
(0.025)\end{array}$ & $\begin{array}{c}C>O \\
(0.0018)\end{array}$ & $\begin{array}{l}C>O \\
(0.316)\end{array}$ \\
\hline Transversal & \multicolumn{2}{|c|}{$\bar{L}$} & $l$ & $\sigma_{L}$ & $\sigma_{l}$ & $N_{p}$ & $N_{e}$ \\
\hline Osteoporotic & \multicolumn{2}{|c|}{ [3.87 5.59$]$} & {$\left[\begin{array}{lll}0.33 & 1.06\end{array}\right.$} & {$\left[\begin{array}{lll}1.25 & 3.02\end{array}\right]$} & {$\left[\begin{array}{ll}0.66 & 1.13\end{array}\right]$} & [3023 4723] & 17 79] \\
\hline Control & \multicolumn{2}{|c|}{$\left[\begin{array}{ll}4.20 & 5.33\end{array}\right]$} & {$\left[\begin{array}{ll}0.5 & 0.99\end{array}\right]$} & {$\left[\begin{array}{lll}1.53 & 2.81\end{array}\right]$} & {$\left[\begin{array}{ll}0.74 & 1.12\end{array}\right]$} & {$\left[\begin{array}{ll}3501 & 4383\end{array}\right]$} & $2364]$ \\
\hline $\begin{array}{c}\text { Wilcoxon Test } \\
\text { (p-value) }\end{array}$ & \multicolumn{2}{|c|}{$\begin{array}{c}C>O \\
(0.13)\end{array}$} & $\begin{array}{l}C>O \\
(0.035)\end{array}$ & $\begin{array}{l}C>O \\
(0.137) \\
\end{array}$ & $\begin{array}{l}C>O \\
(0.056)\end{array}$ & $\begin{array}{l}C>O \\
(0.46)\end{array}$ & $\begin{array}{l}C<O \\
(0.13)\end{array}$ \\
\hline \multicolumn{2}{|c|}{$\begin{array}{c}\text { Global } \\
\text { Osteoporotic }\end{array}$} & & $N_{p}$ & atersections & $N_{p}^{\mathrm{L}} / N_{p}^{\mathrm{T}}$ & \multicolumn{2}{|c|}{$\sigma_{\mathrm{L}}^{2}(\theta) / \sigma_{\mathrm{T}}^{2}(\theta)$} \\
\hline \multicolumn{2}{|c|}{ Osteoporotic } & 128 & 66 18591] & {$\left[\begin{array}{lll}1567 & 2864\end{array}\right]$} & {$\left[\begin{array}{ll}1.01 & 1.56\end{array}\right.$} & \multicolumn{2}{|c|}{$\left[\begin{array}{ll}0.22 & 5.87\end{array}\right]$} \\
\hline \multicolumn{2}{|c|}{ Control } & 140 & 45 16987] & {$\left[\begin{array}{ll}1777 & 2540\end{array}\right]$} & {$\left[\begin{array}{ll}1.08 & 1.54\end{array}\right]$} & \multicolumn{2}{|c|}{$\left[\begin{array}{lll}0.30 & 4.50\end{array}\right]$} \\
\hline \multicolumn{2}{|c|}{$\begin{array}{c}\text { Wilcoxon Test } \\
\text { (p-value) }\end{array}$} & & $\begin{array}{l}<O \\
(0.01)\end{array}$ & $\begin{array}{l}C<O \\
(0.009)\end{array}$ & $\begin{array}{c}C>O \\
(0.12)\end{array}$ & \multicolumn{2}{|c|}{$\begin{array}{l}C<O \\
(0.032)\end{array}$} \\
\hline
\end{tabular}

information given by our features added to BMD and Age. From the analysis of the figure (2]a), we obtain three groups of features weakly correlated. The first, strongly correlated to the first factorial axis, contains length $\bar{L}$, the half-width $\bar{l}$ of the projected trabeculae, their variance for the two directions $\left(\bar{L}(L), \bar{L}(T), \sigma_{\bar{L}}(L), \sigma_{\bar{L}}(T), \bar{l}(L), \bar{l}(T), \sigma_{l}(L), \sigma_{\bar{l}}(T)\right)$ and the number of intersections. The second, describing the second factorial axis, contains features deduced from the numbers of pixels $\left(N_{p}(L), N_{p}(T), N_{p}(L) / N_{p}(T)\right)$. The last group including BMD and age is not well depicted in this first factorial plane.

The projection of our sample on the first factorial plane shows the pertinence of the first principal component ("facteur 1" in figure (2.a), to discriminate between OP and CP. We have chosen to separate the osteoporotic with vertebral fracture (black circles) from those with other fractures (darck grey circles) in addition. The discrimination between $\mathrm{OP}$ and $\mathrm{CP}$ is more striking when we limit ourselves to $\mathrm{OP}$ with vertebral fracture.

To classify OP and CP, logistic regression models were applied. The best models with three features were obtained as follows : one feature of first group explaining the spread of our sample was entered into the model combined with $N_{e}(T)$ and BMD. With $\bar{l}(T)$ as the feature of the first group, we obtained $77 \%$ well classified with $89.7 \%$ in the CP class and a very good fit with a smalle p-value $\left(\leq 10^{-3}\right)$. 


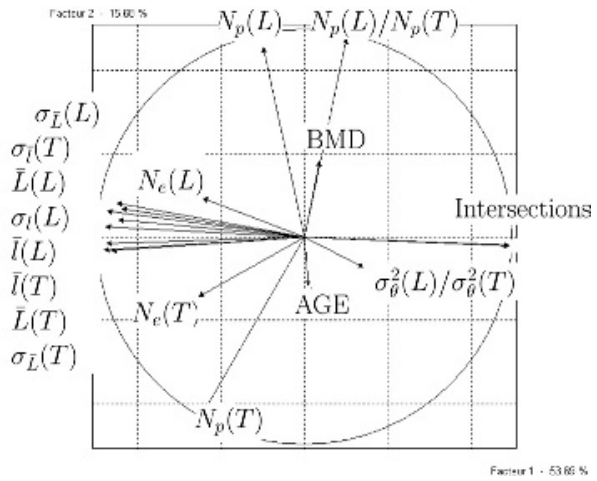

(a)

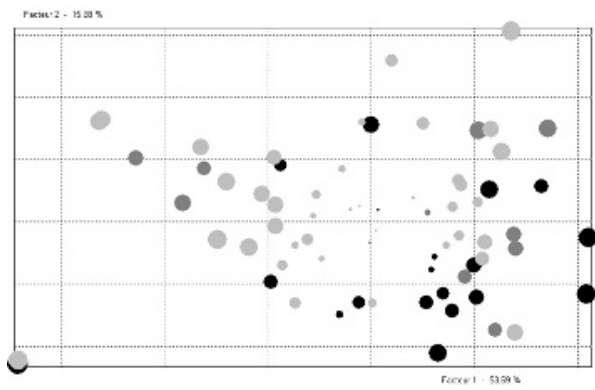

(b)

Fig. 2. The figure (a) is the correlation circle associated with the first factorial plane of the morphological features combined with bone density BMD and AGE. The figure (b) is the projection of our sample on this plane. The discs of black color give the position of OP having a vertebral fracture. The discs of dark grey color are patients having both vertebral fracture and another one fracture. Finally, the discs of light grey color give the position of CP. Bigger is the circle, higher is the proximity to the plane.

\section{Conclusion and Perspectives}

In this paper, we have extracted features from calcaneus radiographs that reflect the trabeculae microarchitecture of the bone. Statistical tests have indicated that the inter and intra projected distances are key-discriminant features. In fact, the results obtained are promising in the sense that :

- additional information was producted with respect to BMD and age,

- they permitted to point out well known osteoporosis symptoms related to the microarchitecture from a radiograph instead of an invasive test.

So, larger data set and more sophisticated methods should be used in order to consider more than two classes. Then it could be possible to study separately different kind of fractures.

\section{References}

1. Consensus development Conference: diagnosis, prophylaxis, and treatment of osteoporosis, Amer. J. Med., vol. 94, no. 6, pp. 646-650, June 1993.

2. S.A. Goldstein, "The mechanical properties of trabecular bone: Dependence on anatomic location and function," J. Biomech., vol. 20, pp. 1055-1061, 1987.

3. S. Majumdar, D. Newitt, M. Jergas, A. Gies, et al., "Evaluation of technical factors affecting the quantification of trabecular bone structure using magnetic resonance imaging," Bone, vol. 17, pp. 417-430, 1995.

4. V. Paquet, P. Battut, H.V. Blanc D. Ferrand, "On the use of grey run lenth matrices in trabecular bone analysis," Proc. of the Conf. on the Image Proc. and its Applic., pp. 445-449, 4-6 July 1995. 
5. T. Lundahl, W.J. Ohley, S.M. Kay, R. Siffert, "Fractional Brownian motion: a maximum likelihood estimator and its application to image texture," IEEE Trans. Med. Imag., vol. MI-5, no. 3, pp. 152-161, September 1986.

6. C.L. Benhamou, R. Harba, E. Lespessailles, E. Jacquet, D. Toulière, R. Jennane, “ Fractal organisation of trabecular bone images on calcaneus radiographs," J. Bone Mineral Res., vol. 9, pp. 1909-1918, 1994.

7. T.E. Southard, K.A. Southard, "Detection of simulated osteoporosis in maxillae using radiographic texture analysis," IEEE Trans. on Biomed. Eng., vol. 43, no. 2, pp. 123-132, February 1996.

8. J.S. Gregory, R.M. Junold, P.E. Undrill, R.M. Aspden, "Analysis of trabecular bone structure using Fourier transforms and neural networks," IEEE Trans. on Inf. Tech. in Biomed., vol. 3, no. 4, pp. 289-294, December 1999.

9. W.G.M. Geraets, "Computer-aided analysis of the radiographic trabecular pattern," Ph. D. Thesis, Netherlands.

10. C. Arcelli, G. Ramella, "Finding grey skeletons by iterated pixel removal," Image and Vision Computing, vol. 13, no. 3, pp. 159-267, April 1995.

11. S. Chen, F.Y. Shih, "Skeletonization for fuzzy degraded character images," IEEE Trans. on Image Proc., vol. 5, no. 10, pp. 1481-1485, October 1996.

12. S. S. Mersal, A.M. Darwish, "A new parallel thinning algorithm for gray scale images," IEEE Nonlinear Signal and Image Proc. Conf., Antalya, Turkey, June 1999.

13. S. Sevestre-Ghalila, A. Benazza-Benyahia, H. Cherif, W. Souid, "Texture analysis for osteoporosis detection with morphological tools," Medical Imaging 2001, SPIE Conf., Milan Sonka, Kenneth M. Hanson Eds., vol. 4322, pp. 1534-1541, San Diego, California, USA, 17-23 February 2001.

14. C. Steger, Unbiased extraction of curvilinear structures from $2 D$ and $3 D$ images, PhD Thesis, Technical University of Munchen, 1998.

15. P. Banerji, S.G. Kabra, "The trabecular pattern of calcaneus as an index of osteoporosis" Journal British Editorial Society of Bonne and joint Surgery vol 65-B, no. 2, pp. 195-198, 1983.

16. T. Koller, C. Grieg, G. Szekély, D. Dettwiler, "Multiscale detection of curvilinear structures in 2D and 3D image data," Proc. of the Fifth Internat. Conf. on Computer Vision, pp. 864-869, 1995. 